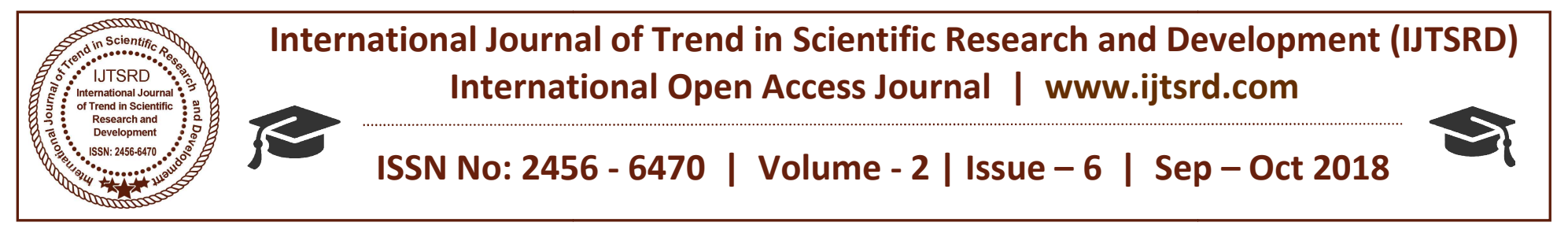

\title{
Wireless Body Area Network: A Review on Issues, Routing Techniques and Various Applications
}

\author{
Madhukar Anand ${ }^{1}$, Ravi Kumar Malik ${ }^{2}$ \\ ${ }^{1}$ Research Scholar, ${ }^{2}$ Assistant Professor \\ Department of ECE, Geeta Engineering College, Panipat \\ Kurukshetra University, Kurukshetra, Haryana, India
}

\begin{abstract}
Advancements in recent wireless technology, integrated circuit, electronic devices and autonomous sensing of physiological parameters, gave the birth of human monitoring system or short range wireless communication network, called Wireless Body Area Networks (WBANs). It is one of the major applications of Wireless Sensor Networks (WSNs), used in medical and as well as non-medical field. In WBAN, various tiny, intelligent, low powered and invasive/non-invasive sensor nodes are placed inside or outside the human body to collect the data. These sensor nodes are referred to as independent device with limited communication capabilities. Deployed sensor nodes are intended to early detection of various types of life threatening diseases viz. cancer, asthma etc., using different types of biomedical sensor nodes like ECG (Electrocardiogram), EEG (Electroencephalogram), pulse rate, temperature, blood pressure etc.
\end{abstract}

Keyword: WBAN, patient monitoring, routing, WBAN applications

\section{INTRODUCTION}

According to IEEE 802.15.6, WBAN is defined as "a communication standard optimized for low power devices and operation on, in or around the human body to serve a variety of applications including medical and non-medical field". It adopted the WBANs as the next generation of wireless technology for Wireless Personal Area Networks (WPANs). A Task group of WPANs has worked since November, 2007 to standardize the WBANs technology [1]. The transmission rate of WBANs is $10 \mathrm{Kbps}$ to $10 \mathrm{Mbps}$, which distinguish the WBANs from the existing WPANs.

Requirements of IEEE 802.15.6 standard are listed below [2]:

i. Packet error rate (PER) should be less than $10 \%$ for 256 octet payload for a majority (95\%) of the best performing links based on PER.

ii. Nodes should be capable of being removed and added to the network in less than 3 seconds.

iii. Jitter, latency and reliability should be supported for WBAN applications that require them. Latency should be less than $125 \mathrm{~ms}$ in medical applications and less than $250 \mathrm{~ms}$ in non-medical application, while latency should be less than $50 \mathrm{~ms}$.

iv. On-body and in-body WBANs should be capable of coexisting within range.

v. Maximum radiation transmission power should be less than $1 \mathrm{~mW}(0 \mathrm{dBm})$.

vi. WBANs should be capable of operating in heterogeneous environment.

vii. WBANs must incorporate QoS management features to be self-healing and secure as well as allowing priority services.

viii. Transmission range of WBANs is up to 3 meters.

ix. Range of data rates of sensor nodes is from $1 \mathrm{Kbit} / \mathrm{s}$ to $10 \mathrm{Mbit} / \mathrm{s}$.

\section{ROUTING PROTOCOL DESIGN ISSUES}

Since, WBAN is a subset of Wireless Sensor network, in spite of that protocols used in WSNs are not directly used by WBANs due to its some specific circumstances like architecture, density, data rate, latency and mobility requirements [3]. Precise requirement of WBANs impose certain constraints on the design of their routing protocol. Each protocol that is designed for WBANs only tries to satisfy a 
specific requirement. Not a single protocol satisfies the requirements of more than one application [4]. Here, we have discussed few factors which make it difficult to choose a routing protocol for WBANs.

i. Mobility: Due to complex structure and movement of body, network topology is changed and routes among nodes are distorted. Possibility of data loss and signal blockage is high. Therefore, WBANs require an adaptive routing algorithm that adapts posture movement of human body [4].

ii. Efficient transmission range: Low radio frequency transmission range leads to disconnection and frequent partitioning of link among sensor nodes in WBANs. If the range of remitting data is less than the threshold value, then choice to select a new route for next sensor node is reduced. Also the fewer the number of neighbors, the lower the probability of packets to arrive at the destination within a certain hop count [5].

iii. Quality of Services: It deals with the critical data (EEG, ECG), reliable sensitive data and ordinary data of the body. The proposed protocol should be aware of these types of data [6].

iv. Security \& Privacy: Due to energy constraints, it is impossible to apply the conventional techniques of privacy and security. So, a routing protocol is to be deal with privacy and security of patients' data [6].

v. Energy consumption: Continuous data sensing and transmission, and greater distance between communicating nodes are the main factors of more energy consumption. As the nodes are placed inside or outside the human body therefore, batteries of nodes can't frequently recharge or replaced. The sensor nodes have to transmit, receive and sense the data which might result in quick energy depletion of nodes [7].

vi. Heterogeneous Environment: Different-different nodes are implanted in or on the body to detect the different types of diseases. Each sensor node is heterogeneous in nature, because memory and power consumption are varied from one to another [7].

vii. Electromagnetic Radiation: Heat generated by sensors affect the sensitive organs of human body and also damages the tissues. The transmission power of sensor nodes should be low. Researchers need to careful to keep human tissues safe from overheating caused by absorption of electromagnetic radiation [8].

viii. Global Network Lifetime: Network lifetime is referred to as the time interval from the network starts to the time of network destroyed, which leads to the network partitioning. Since it is not easy to recharge or replace the battery frequently, network lifetime is one of the most important for WBANs as compared to WPANs and WSNs [8].

ix. Limitations of resources: Data capacity, energy and unstable device in WBANs are strictly limited. Due to limitation of these available resources, nodes stopped their work due to unavailable battery power, memory and bandwidth limitations, which are major threats to QoS [8].

\section{Classification of WBANs Routing Protocol}

A variety of routing protocols is to be proposed for WSNs, MANET and WBANs. WBANs are similar to MANET in terms of moving topology with groupbased movement rather than node based movement [9]. Though, sensor nodes are deployed in WBANs with limited battery power. As a result, it is essential for WBANs to have a longer network lifetime to avoid regular recharging and replacement of nodes attached to the patients\persons. These all things should be kept in mind, when a routing protocol is to be design. Routing protocols in WBANs is classified on the basis of different parameters like QoS aware routing, temperature aware routing, cluster based routing, cross layer routing, opportunistic routing etc. [9]. Knowledge of various types of routing protocol is very important because they can affect the performance of network. Figure 1 describes the classification of WBANs' routing protocol.

Protocols of WBAN

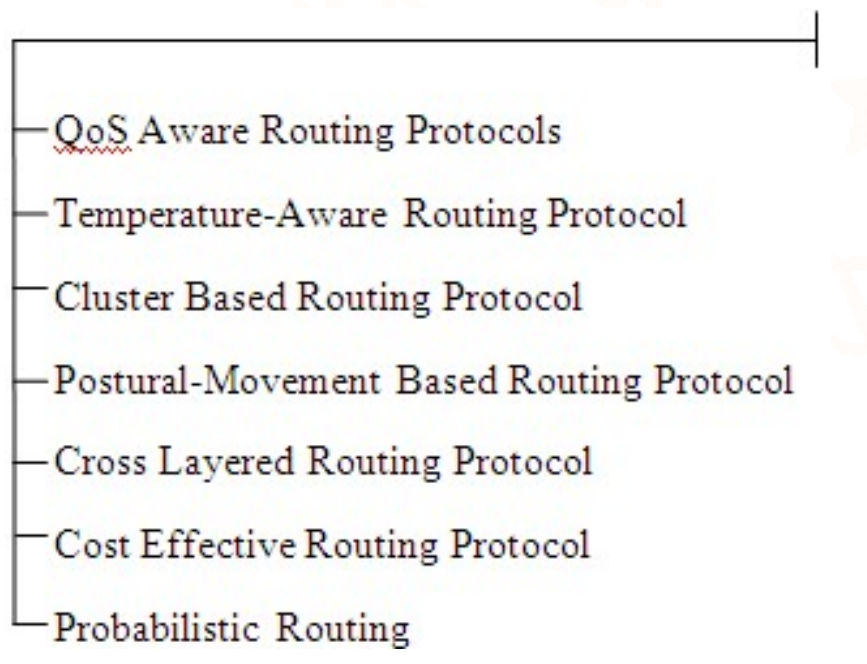


Figure 1: Routing Strategies of WBANs

i. QoS aware routing protocols: It use differentdifferent modules for providing different QoS metrics. The modules used in this routing are: the power-efficiency module, the reliabilitysensitive module, the delay-sensitive module and the neighbor manager. For this reason, they provide high reliability, lower end to end delay and higher packet delivery ratio. It is very difficult to design a QoS aware routing protocol, using variety of modules. These routing protocols are mainly suffering from high complexity [5].

ii. Temperature aware routing protocols: Mainly designed to minimize the temperature arise in body due to sensor nodes, which damage the surrounding tissues and sensitive organs of human body by reducing the blood flow, growth of bacteria, enzymatic reaction etc. A routing protocol of WBANs should be aware about electromagnetic radiation. The main of these type of routing protocol is to detect hot-spot link and far away from the body. However, these protocols suffer from system complexity and overhead of nodes [6].

iii. Cluster based routing protocol: Nodes are divided into one or more than one cluster. A cluster head is chosen for each group of cluster. Data are transmitted via an intermediate node called forwarder node, parent node or cluster head. Different approaches are proposed to select the cluster head in WBANs by various authors. The cluster head is intended to reduce the direct communication between sensors or nodes to the sink node. The major problem with cluster based routing is, it takes a lot of time to choose the cluster head in each round [7].

iv. Postural movement based topology: Due to body posture movement, links between nodes of WBAN are partitioned or disconnected. This type of problem is solved by defining of cost function, which is updated periodically. This type of protocol chooses a route from sensors to sink node. E.g.: On-Body store and Flood routing, Energy efficient \& thermal aware routing [8].

v. Cross layered routing: It builds a spanning tree and TDMA time slots are assigned in a distributed manner. Its aim to improve the overall performance of WBANs and achieve high throughput, low energy consumption by solving the network and MAC layer issues. They can't provide better performance in case of mobility and require large amount of overhead to exchange the information in the network [9].

vi. Cost effective routing: These routing protocols updates a cost function based on effective information in every round, and select a route with minimum or maximum cost function. A large number of transmissions are required to update the information of cost function [10].

vii. Probabilistic routing protocols: They update their cost function periodically, based on link state information. A path is established with minimum cost function. This type of routing protocol requires a large number of transmissions to update the link-state information [11].

\section{Applications of WBANs}

The main objective behind WBANs is to improve the quality of life of human being. And that's why; it is not only restricted to medical application. It spans a wide area of medical and non-medical applications from sports and entertainment to ubiquitous health care, military and many more. In sports, sensor nodes are deployed on the player to check the level of lactic acid in blood [1]. As today, internet has changed the thinking of common people, in the same way it is expected that WBANs will change the life style of each and every person by the payback of this technological advancement in maximum number of applications [9]. Figure 2 shows the applications of WBANs in medical and as well as in non-medical field. 


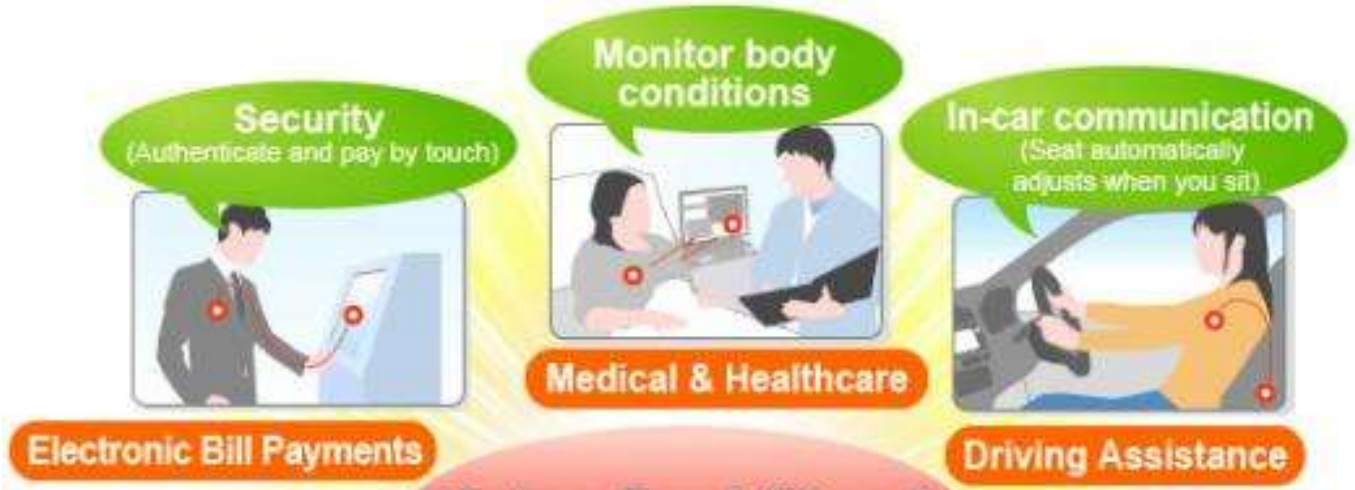

Various Capabilities of

Body Area Networks

Ofifice Security

\subsection{Medical Application}

Advancement in miniaturization of small biomedical sensor nodes, gave a new opportunity to monitor the health condition remotely [10]. These sensor nodes collect the large amount of data automatically, reducing the cost and inconvenience of regular visits to physician. To achieve these things, different types of sensor nodes are used to detect the different types of diseases like cancer detection, Asthma etc. Description about various types of sensors is given in next section.

1. Sleep Staging: Nowadays, there are thousands of people who are facing sleeplessness hitch. It is scientifically proved that if a person will not sleep properly, it can affect their health too. Due to this reason, sometimes physician wants to monitor the sleeping time of patients, which is quite difficult before WBANs. Now, it is easy \& comfortable by implanting the sensor nodes on the human body [10].

2. Military and Defense: Network enabled capability is a program running in our military and defense organization, which uses the advanced information system to achieve more effective military results. Sensor nodes can be used both, at an individual level for a soldier or for whole squad. At an individual level, soldiers fatigue can be monitored using the different parameters like heart-beat and blood-pressure measures sent through sensor nodes which are worn by them [11].

3. Cancer Detection: Events which require prediagnosis like tumors and cancer causing cells can be detected using WBANs with diffusion based molecular- communication. In this type of communication, it is possible to deal with fatal situation for diagnosing and destroying cancer using tumors [12].

4. Cardiovascular Diseases: These types of diseases are the source of number of deaths each year worldwide. This ratio can be decreased by a big amount by analyzing the waveforms of cardiosignals collection, which provides better patient monitoring and early detection of symptoms of diseases [12]

5. Asthma: WBANs provide a real time event of the presence of harmful substances in the air and then informed the current status to the physician and concerned people. So, the asthmatic person will be alerted in advance and it can save lives of many people [13].

6. Life Shirt: It is a comfortable and non-invasive "smart-garment" that collects the data during a patient's daily routine and provides the current status of data to the physician remotely. It uses 
integrated sensor nodes including respiratory bands and an ECG sensor node. It also tracks and records the posture and physical activity of body [10].

7. eWatch: It can be used for applications such as context aware notification, elderly monitoring and fall detection. A eWatch system can sense if the person is in trouble and then query to confirm that is an emergency [13].

8. Artificial Retina: WBANs can assist those people who lost their vision, by using retina prosthesis chip implanted within a human eye as shown in figure 3.

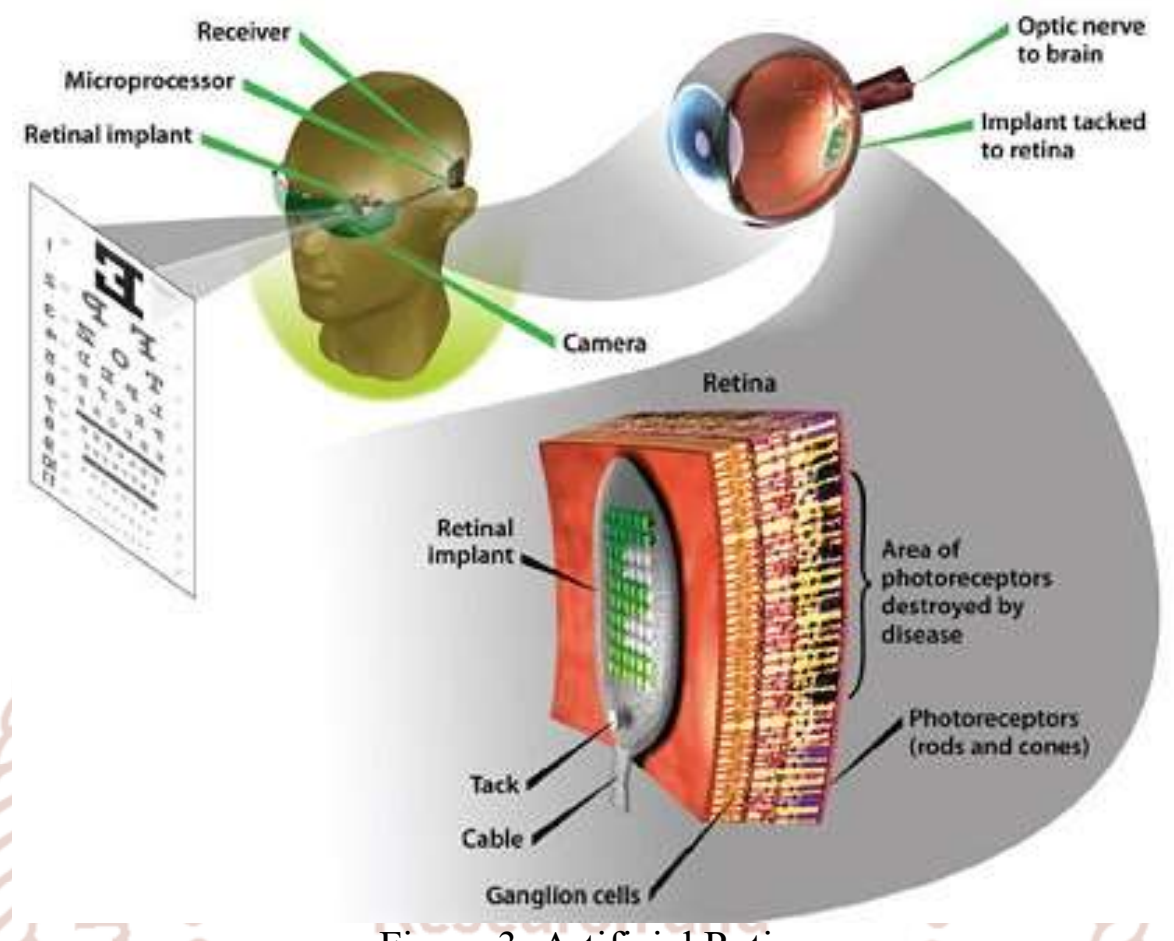

Figure 3: Artificial Retina

9. Diabetes Control: Glucose level monitor require bit rate of less than $1 \mathrm{kbps}$ and automatic drug delivery requires a bit rate of approximately less than $16 \mathrm{kbps}$. WBANs have been enabled with diffusion based molecular communication, which can be used in developing automatic drug delivery systems. In case of this, system will automatically analyze the user's data based on different parameters, and automatically prescribed the medicine in case of emergency [11].

\subsection{Non-Medical Application}

The aging population, increasing cost in formal health care and the most important thing is, the individual place on living independently, all motivate the development of innovative-assisted living technologies for safe and independent aging [11]. Non-medical applications of WBANs, improve the quality of life in order to maintain a more independent lifestyle using home automation and other. Assisted livings facilities have emerged have an alternative facility for people with disability and elderly, who are not considered independent but do not need around the clock medical care, as in retirement homes.

4.2.1 Real Time Streaming: It involves video streaming such as capturing a video clip by the camera in a cellular phone, trading shows for sport goods along with the latest fashion design and 3D videos. This category also includes stream transfer, which is used for remote control of entertainment devices, body gesture recognition or motion capture, vital sign and body information-based entertainment service, identification, emotion detection and to monitor forgotten things by sending an alert o the owner [12].

4.2.2 Entertainment Applications: It consists of gaming application and social networking. Appliances such as microphones, MP3-players, cameras, headmounted displays and advanced computer appliances can be used as devices integrated in WBANs. They can be used for gaming purpose (game control with hand gesture, mobile body motion game and virtual world game), personnel item tracking, exchanging 
International Journal of Trend in Scientific Research and Development (IJTSRD) ISSN: 2456-6470

digital profile or business cards and consumer electronics [10].

4.2.3 Emergency (Non-Medical): Off-body sensor nodes are used to detect a non-medical emergency such as, fire in the home or flammable/poisonous gas in the house and just inform to the body worn devices to warn the wearer of emergency condition [12].

4.2.4 Games or sports: Interactive game is a growing application of WBANs. As the players move their limbs, deployed sensor nodes detects the movement and send data to the gaming device. Muscles fatigue is also to be measured by sensing the accumulation of lactic acid in muscles [11].

4.2.5 Secure Authentication: Due to duplicability and forgery, secure authentication is one of the most important non-medical applications of WBANs. It concerns with the utilizing physiological and behavioral biometrics such as iris recognition, finger prints and facial patterns [13].

\section{CONCLUSION}

WBANs are mainly designed to improve quality of life of the human being. Reliable communication using multi-hop topology or via cluster head with minimum energy consumptions is one of the major issues in WBANs. Due to this reason, an efficient routing protocol is to be needed. As the routing play an important role in WBANs to maintain the delivery of patients' data on time. Electromagnetic radiation, signal fading, packet delivery ratio and stability period of network are also the major concerns in WBANs. Signal fading leads to increase delay and decrease the throughput of the network. A selfadapting routing protocol is required to find an optimal path in multi-hop routing or cluster based routing, in case of dead node. Task performed by sensor nodes and their configuration are the two things, which should be kept in mind at the design of routing protocol. Hence, aspire of this paper is to understand the different types of routing techniques in WBANs and propose an optimal routing technique for WBANs, in terms of throughput and stability period.

\section{REFERENCES}

1. K. A. Khaliq, O. Chughtai, A. Qayyum and J. Pannek, "An emergency alert system for elderly/special people using VANET and WBAN," 2017 13th International Conference on
Emerging Technologies (ICET), Islamabad, 2017, pp. 1-6.

2. D. Liu, J. Wang, C. Jiang, F. Ren and Y. Ren, "A contention-oriented node sleeping MAC protocol for WBAN," 2018 IEEE Wireless Communications and Networking Conference (WCNC), Barcelona, 2018, pp. 1-6.

3. N. Santos N. Santos and S. Motoyama S. Motoyam, "A Large Scale Platform Using WBAN Technology for Patient Monitoring," in IEEE Latin America Transactions, vol. 16, no. 3, pp. 705-711, March 2018.

4. T. Manna and I. S. Misra, "Implementation of energy efficient WBAN using IEEE 802.15.6 scheduled access MAC for e-healthcare," 2018 10th International Conference on Communication Systems \& Networks (COMSNETS), Bengaluru, 2018, pp. 267-274.

5. S. M. Demir, F. Al-Turjman and A. Muhtaroğlu, "Energy Scavenging Methods for WBAN Applications: A Review," in IEEE Sensors Journal, vol. 18, no. 16, pp. 6477-6488, Aug.15, 152018.

6. R. Saha, S. Biswas and G. Pradhan, "A priority based routing protocol with extensive survey and comparison of related works for healthcare applications using WBAN," 2017 International Conference on Wireless Communications, Signal Processing and Networking (WiSPNET), Chennai, 2017, pp. 1424-1430.

7. Y. Zhang, C. Li, T. H. Luan, Y. Song and X. Yuan, "Prototype System Based Enhanced Scheduled Access Mechanism for WBAN," 2017 IEEE 86th Vehicular Technology Conference (VTC-Fall), Toronto, ON, 2017, pp. 1-5.

8. L. Wang, F. Hu, Z. Ling and B. Wang, "Wireless Information and Power Transfer to Maximize Information Throughput in WBAN," in IEEE Internet of Things Journal, vol. 4, no. 5, pp. 16631670, Oct. 2017.

9. F. Sun, C. Yi and Y. Li, "Battery-friendly scheduling policy in MAC layer for WBAN data packets transmission," in IET Communications, vol. 11, no. 9, pp. 1423-1430, 6222017.

10. F. Hu, X. Liu, M. Shao, D. Sui and L. Wang, "Wireless Energy and Information Transfer in WBAN: An Overview," in IEEE Network, vol. 31, no. 3, pp. 90-96, May/June 2017. 
International Journal of Trend in Scientific Research and Development (IJTSRD) ISSN: 2456-6470

11. X. Yang, L. Wang and Z. Zhang, "Wireless Body Area Networks MAC Protocol For Energy Efficiency and Extending Lifetime," in IEEE Sensors Letters, vol. 2, no. 1, pp. 1-4, March 2018, Art no. 7500404.

12. D. Sui, F. Hu, W. Zhou, M. Shao and M. Chen, "Relay Selection for Radio Frequency EnergyHarvesting Wireless Body Area Network With
Buffer," in IEEE Internet of Things Journal, vol. 5, no. 2, pp. 1100-1107, April 2018.

13. D. Fernandes, A. G. Ferreira, R. Abrishambaf, J. Mendes and J. Cabral, "A Low Traffic Overhead Transmission Power Control for Wireless Body Area Networks," in IEEE Sensors Journal, vol. 18, no. 3, pp. 1301-1313, 1 Feb.1, 2018.

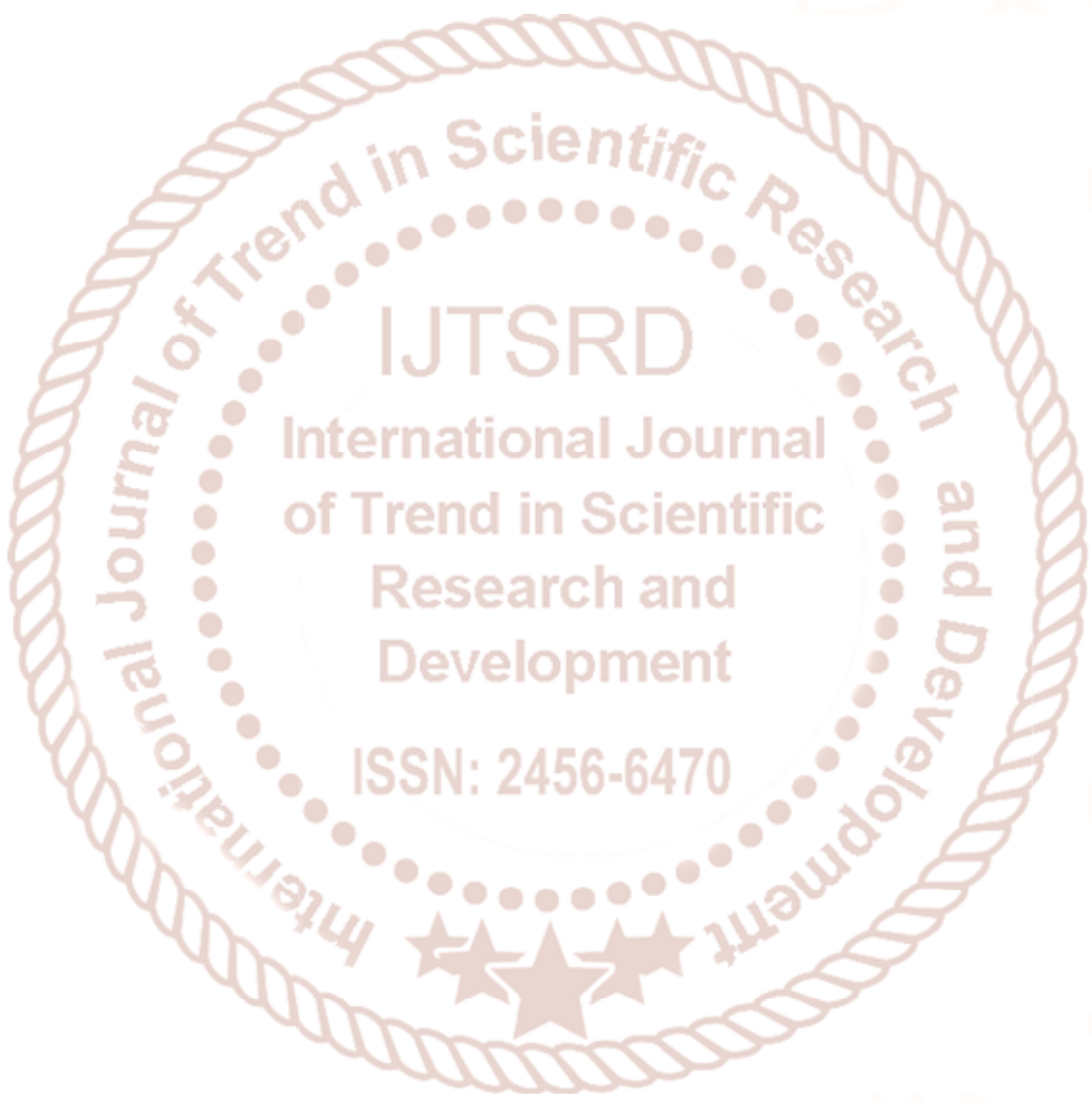

